\title{
Incisional Hernia Post Laparotomy-Incidence and Risk Factors
}

\author{
Amjad Shah ${ }^{1,}$ *, Zia Aftab ${ }^{1}$, Syed Muhammad Ali ${ }^{1}$, Salah Gehani ${ }^{1}$, Khalid Ahmed ${ }^{2}$, \\ Ahmed Almodaris ${ }^{1}$, Rashad Fouad ${ }^{1}$, Abdulrahman Al-Aal ${ }^{1}$ \\ ${ }^{1}$ General Surgery, Department of Surgery, Hamad General Hospital, Doha, Qatar \\ ${ }^{2}$ Trauma and Critical Care, Hamad General Hospital, Doha, Qatar
}

\section{Email address:}

amjad.alishah@gmail.com (A. Shah)

${ }^{*}$ Corresponding author

\section{To cite this article:}

Amjad Shah, Zia Aftab, Syed Muhammad Ali, Salah Gehani, Khalid Ahmed Fellow, Ahmed Almodaris, Rashad Fouad, Abdulrahman AlAal. Incisional Hernia Post Laparotomy-Incidence and Risk Factors. Journal of Surgery. Vol. 6, No. 1, 2018, pp. 19-22.

doi: $10.11648 /$ j.js. 20180601.14

Received: November 27, 2017; Accepted: December 8, 2017; Published: January 16, 2018

\begin{abstract}
Laparotomy incisions are one of the most common procedures performed in any surgical service. However, they carry certain risks like surgical site infections, wound dehiscence and incisional hernia. There have been various risk factors associated with the incidence of incisional hernia post laparotomy. Some of these factors are patients- related like history of diabetes, obesity, smoking, sex and age. The other factors are related to the disease process itself like emergency surgeries, presence of peritonitis, history of radiation to the abdominal cavity or presence of surgical site infection. Another set of factors relates purely with the technique used to close the wound, choice of suture material and expertise of the surgeon. Methods. This is a retrospective chart review. Data was collected from all patients who were previously admitted at Hamad General Hospital for laparotomy and subsequently developed incisional hernia. Methods. All patients of age 18 years or above who were found to have incisional hernia after laparotomy were included in the study. Hospital medical records database were used for file reviews. Patients characteristics like age, sex, obesity, history of smoking, DM etc were recorded. Nature of surgeries like emergency or elective were documented along with other factors like suturing techniques etc. Results. The total number of study subjects was 672, out of which $47(6.9 \%)$ developed incisional hernia. Out of these 23 were male and 24 were female. Diabetes was identified in 18 patients (38.3\%) whereas the incidence of smoking, steroid use, COPD and obesity was $2(4.3 \%)$, $0(\%), 3(6.4 \%)$ and $7(14.9 \%)$ respectively. Other risk factors like the nature of surgery (emergency vs electives) were addressed also. The incidence of immediate postop complications was also addressed. Three patients out of $47(6.4 \%)$ developed surgical site infection whereas one patient $(2.1 \%)$ had post -op wound dehiscence. The number of contaminated and dirty wounds during the initial surgery was $10(21.3 \%)$ and $6(12.8 \%)$ respectively.
\end{abstract}

Keywords: Laparotomy, Incisional Hernia, Risk Factors, Incidence

\section{Introduction}

Laparotomy incision has been the standard for abdominal surgeries. It gives better and easy access to the abdominal cavity. However, it carries certain risks like surgical site infections, wound dehiscence and incisional hernia $[1,2,7]$. There have been various risk factors associated with the incidence of incisional hernia after laparotomy. Some of these factors are patients- related like history of diabetes, obesity, smoking, sex and age $[3,5,9]$. The other factors are related to the disease process itself like emergency surgeries, presence of peritonitis, history of radiation to the abdominal cavity or presence of surgical site infection $[2,3,8]$.

Incisional hernia is one of the most common complications after laparotomy. The reported incidence is highly variable depending on underlying disease, type of incision used, length of follow up and method for hernia detection. Numerous risk factors associated with an increased incisional 
hernia incidence have been reported, including gender (both male and female), age, surgical site infection, obesity and aortic aneurysm $[1,4,11,6]$. Another set of factors relates purely with the technique used to close the wound, choice of suture material and expertise of the surgeon [2, 4, 7, 11].

Research studies have been conducted before aiming at the potential risk factors for the development of incisional hernia after laparotomies. Millburn D et al, studied the effect of stitch length on the incidence of surgical site infection, wound dehiscence and incisional hernia after midline laparotomy surgeries. They found out that the incidence of incisional hernia, wound dehiscence and surgical site infection was much lower when the stitches were placed near to the wound edge $(5-8 \mathrm{~mm})$ than long traditional stiches which are paced more than $1 \mathrm{~cm}$ from the wound edge [2]. In the same study male sex, obesity, diabetes, presence of wound contamination were found to have as independent risk factors for the incidence of incisional hernia [2].

Incisional hernia can be a challenging clinical problem especially in morbidly obese patients, patients who have diabetes and who are steroid users. Other researchers have studied the clinical impact of morbid obesity and steroid use on the incidence of incisional hernia and found to have a protective effect of polypropylene mesh on the incidence of incisional hernia $[4,9,11]$.

\section{Materials and Methods}

\subsection{Data Source and Study Population}

Data was collected from the hospital patients' database from the medical records office.

Hospital medical records database were used for file reviews. Patient's characteristics like age, sex, obesity, history of smoking, DM etc. were recorded. Nature of surgery, like emergency or elective was documented along with other factors like suturing techniques etc.

\subsection{Inclusion Criteria}

All patients aged 18 years or above who were previously admitted to our hospital for abdominal surgery and subsequently developed incisional hernia after laparotomy incisions.

\subsection{Exclusion Criteria}

Patients below 18 years were excluded.

\section{Data Analysis}

Data obtained was compiled in a systemic way and analyzed by using SPSS database for the presence of different risk factors.

Table 1. Patient Demographics and Characteristics of Surgery.

\begin{tabular}{ll}
\hline Demographic & \\
Age (years, M (SD)) & $53.22(15.22)$ \\
Type of surgery N (\%) & $23(48.9)$ \\
Emergency & $24(51.1)$ \\
Elective & $9(19.1)$ \\
Wound Condition N (\%) & $22(46.8)$ \\
Clean & $10(21.3)$ \\
Clean contaminated & $6(12.8)$ \\
Contaminated & \\
Dirty & $43(91.5)$ \\
Post op complications N (\%) & $3(6.4)$ \\
None & $1(2.1)$ \\
SSI & \\
Wound dehiscence & \\
\hline
\end{tabular}

Table 2. Incidence of risk factor variables.

\begin{tabular}{|c|c|c|c|c|c|}
\hline \multicolumn{6}{|l|}{ SEX } \\
\hline & & Frequency & Percent & Valid Percent & Cumulative Percent \\
\hline \multirow{3}{*}{ Valid } & $\mathrm{F}$ & 24 & 51.1 & 51.1 & 51.1 \\
\hline & M & 23 & 48.9 & 48.9 & 100.0 \\
\hline & Total & 47 & 100.0 & 100.0 & \\
\hline \multicolumn{6}{|c|}{ Diabetes } \\
\hline & & Frequency & Percent & Valid Percent & Cumulative Percent \\
\hline \multirow{3}{*}{ Valid } & 0 & 29 & 61.7 & 61.7 & 61.7 \\
\hline & 1.0 & 18 & 38.3 & 38.3 & 100.0 \\
\hline & Total & 47 & 100.0 & 100.0 & \\
\hline \multicolumn{6}{|c|}{ smoking } \\
\hline & & Frequency & Percent & Valid Percent & Cumulative Percent \\
\hline \multirow{3}{*}{ Valid } & 0 & 45 & 95.7 & 95.7 & 95.7 \\
\hline & 1.0 & 2 & 4.3 & 4.3 & 100.0 \\
\hline & Total & 47 & 100.0 & 100.0 & \\
\hline \multicolumn{6}{|c|}{ Steroid use } \\
\hline & & Frequency & Percent & Valid Percent & Cumulative Percent \\
\hline Valid & 0 & 47 & 100.0 & 100.0 & 100.0 \\
\hline
\end{tabular}




\begin{tabular}{|c|c|c|c|c|c|}
\hline \multicolumn{6}{|c|}{ obesity } \\
\hline & & Frequency & Percent & Valid Percent & Cumulative Percent \\
\hline \multirow{3}{*}{ Valid } & 0 & 40 & 85.1 & 85.1 & 85.1 \\
\hline & 1.0 & 7 & 14.9 & 14.9 & 100.0 \\
\hline & Total & 47 & 100.0 & 100.0 & \\
\hline
\end{tabular}

\begin{tabular}{llllll}
\hline COPD & & & & & \\
\hline & & Frequency & Percent & Valid Percent & Cumulative Percent \\
\hline \multirow{3}{*}{ Valid } & 0 & 44 & 93.6 & 93.6 & 93.6 \\
& 1.0 & 3 & 6.4 & 6.4 & 100.0 \\
& Total & 47 & 100.0 & 100.0 & \\
\hline
\end{tabular}

$0=$ absent; $1=$ present .

\section{Results}

The total number of study subjects was 672 out of which $47(6.9 \%)$ developed incisional hernia. Out of those who developed hernia, 23 were male and 24 were female. Diabetes was identified in 18 patients $(38.3 \%)$ whereas the incidence of smoking, steroid use, COPD and obesity was 2 $(4.3 \%), 0(\%), 3(6.4 \%)$ and $7(14.9 \%)$ respectively.

In terms of type of surgery, about half (48.9) were emergency surgeries, while the other half (51.1) were elective. The number of contaminated and dirty wounds during the initial surgery was $10(21.3 \%)$ and $6(12.8 \%)$ respectively. Surgical site infection was encountered in 3 $(6.4 \%)$ cases and wound dehiscence happened in $1(2.1 \%)$ patient.

\section{Discussion}

In this study we report the incidence and risk factors for the incidence of incisional hernia post abdominal surgery. Though the indications for the initial surgery were very varied and the patient population is not homogenous, still there is a great deal of information available from our study.

The total number of study subjects was 672 out of which $47(6.9 \%)$ developed incisional hernia. Out of these 47, 23 were male and 24 were female. Diabetes was identified in 18 patients $(38.3 \%)$ whereas the incidence of smoking, steroid use, COPD and obesity was $2(4.3 \%), 0$ (\%), 3 (6.4\%) and 7 $(14.9 \%)$ respectively.

Other risk factors like the nature of surgery (emergency vs electives) were also addressed.

The incidence of immediate postop complications was also addressed. It is pertinent to note that only three patients out of $47(6.4 \%)$ developed surgical site infection whereas only one patient $(2.1 \%)$ had post -op wound dehiscence. These are major risk factors for the incidence of incisional hernia in other studies so their influence on the incidence of incisional hernia has been kept in check in our institution. This is a significant finding given the number of contaminated and dirty wounds during the initial surgery was 10 (21.3\%) and 6 $(12.8 \%)$ respectively.

The incidence of incisional hernia post abdominal surgery is an important matter which carries health related amplifications for the patients and cost and quality related issues for surgeons and hospitals [8, 12].
Laparotomy incision has been the standard for abdominal surgeries. It gives better and easy access to the abdominal cavity. However, it carries certain risks like surgical site infections, wound dehiscence and incisional hernia. There have been various risk factors associated with the incidence of incisional hernia after midline laparotomy. Some of these factors are patients- related like history of diabetes, obesity, smoking, sex and age. [3, 5,9]. The other factors are related to the disease process itself like emergency surgeries, presence of peritonitis, history of radiation to the abdominal cavity or presence of surgical site infection. [2, 3, 8].

Another set of factors relates purely with the technique used to close the wound, choice of suture material and expertise of the surgeon $[2,4,7,11]$.

Research studies have been conducted before aiming at the potential risk factors for the development of incisional hernia after midline laparotomies. Millburn D et al, studied the effect of stitch length on the incidence of surgical site infection, wound dehiscence and incisional hernia after midline laparotomy surgeries. They found out that the incidence of incisional hernia, wound dehiscence and surgical site infection was much lower when the stitches were placed near to the wound edge $(5-8 \mathrm{~mm})$ than long traditional stiches which are paced more than $1 \mathrm{~cm}$ from the wound edge [2]. In the same study male sex, obesity, diabetes, presence of wound contamination was found to have as independent risk factors for the incidence of in incisional hernia [2].

Studies have also been conducted about the optimum time of surveillance for the occurrence of incisional hernia post abdominal surgery. In one of the studies the authors demonstrated that one year of follow-up is usually not sufficient for the clinical detection of incisional hernia but should be done for at least 3 years $[6,8]$. In our study also the minimum time for occurrence of incisional hernia was reported to be 120 days and the maximum was 4320 days (with a mean of 684 days).

Incisional hernia can be a challenging clinical problem especially in morbidly obese patients, patients who have diabetes and who are steroid users. Other researches have studied the clinical impact of morbid obesity and steroid use on the incidence of incisional hernia and found to have a protective effect of polypropylene mesh on the incidence of incisional hernia $[7,4,11]$. 


\section{Conclusion}

The assessment of risk factors for the incidence of incisional hernia was carried out in this study. Although no risk and effect correlation was carried out in our study, nevertheless this study provides a unique insight into the different risk factors for incidence of incisional hernia in our institution upon which future studies can be based.

\section{References}

[1] Incisional hernia after open resections for colorectal liver Metastases - incidence and risk factors Jan H. Nilsson, Peter Strandberg Holka \& Christian Sturesson HPB (Oxford). 2016 May; 18 (5): 436-441.

[2] Millbourn D, Cengiz Y, Israelsson LA: Effect of stitch length on wound complications after closure of midline incisions: a randomized controlled trial. Arch Surg 2009, 144 (11): 10561059.

[3] Israelsson LA, Jonsson T: Overweight and healing of midline incisions: the importance of suture technique Eur J Surg 1997, 163 (3): $175-180 \mathrm{e}$.

[4] Sugerman HJ, Kellum JM, Reines HD, DeMaria EJ, Newsome HH, Lowry JW: Greater risk of incisional hernia with morbidly obese than steroid-dependent patients and low recurrence with prefascial polypropylene mesh. Am J Surg 1996, 171 (1): 80-84.

[5] Israelsson LA, Jonsson $\mathrm{T}$ : Incisional hernia after midline laparotomy: a prospective study. Eur J Surg 1996, 162 (2): 125-129.

[6] C. Fink P. Baumann, M. N. Wente, P. Knebel, T. Bruckner, A.
Ulrich, J. WernerM. W. Bu" chler and M. K. Diener. Incisional hernia rate 3 years after midline laparotomy Published online 26 November 2013 in Wiley Online Library (www.bjs.co.uk). DOI: 10.1002/bjs.9364.

[7] Ismat M Mutwali, Incisional Hernia: Risk Factors, Incidence, Pathogenesis, Prevention and Complications. Sch. J. App. Med. Sci., 2014; 2 (4E): 1491-1497.

[8] Dunja Kokotovic, MB; Thue Bisgaard, MD, DMSc; Frederik Helgstrand, MD, DMSc. Long-term Recurrence and Complications Associated With Elective Incisional Hernia Repair. JAMA. 2016; $316 \quad$ (15): 1575-1582. doi:10.1001/jama.2016.15217.

[9] René H Forteln Y, Petra Baumann, Wolfgang E Thasler, Markus Albertsmeier, Stefan Riedl, Wolfgang Steurer, Jan Ludolf Kewer and Andreas Shamiyeh. Effect of suture technique on the occurrence of incisional hernia after elective midline abdominal wall closure: study protocol for a randomized controlled trial. Trials 201516: 52.https://doi.org/10.1186/s13063-015-0572-x.

[10] Abd-El-Aal A. Saleem, Hassan A. Abdallah, Osama A. Abdul Raheem, Mohamed A. Yousef. Rate of development of incisional hernia 1 year after urgent midline laparotomy. Al Azhar Assiut Medical Journal 2016, 14: 59-66.

[11] Timothy F. Feldmann, MD, Monica T. Young, MD, and Alessio Pigazzi, MD, PhD Incisional Reinforcement in HighRisk Patients. Clin Colon Rectal Surg. 2014 December; 27 (4): 149-155.

[12] Fischer JP, Basta MN, Mirzabeigi MN, Bauder AR, Fox JP, Drebin JA, Serletti JM, Kovach SJ. A Risk Model and Cost Analysis of Incisional Hernia After Elective, Abdominal Surgery Based Upon 12,373 Cases: The Case for Targeted Prophylactic Intervention. Ann Surg. 